\title{
Criterion for the performance analysis of synchronous and asynchronous sampling instruments based on nonlinear processing
}

\author{
M.A. Catelani \\ G. Iuculano \\ D. Mirri \\ F. Filicori \\ A. Menchetti
}

Indexing terms: Algorithms, Digital instrumentation

Abstract: We propose a criterion for the comparison of different sampling strategies (synchronous, asynchronous and random) and filtering algorithms used in digital instruments which provide the estimate of the time average of a signal processed with a nonlinear conversion of multiple inputs (e.g. wattmeters, RMS voltmeters, ...). This criterion uses the Bayesian approach to incorporate, for every sampling strategy, any prior information on the influences of each incidental quantity which can vary the output of the instrument, transforming this output into a statistic. The asymptotic mean-squared error of the measurements has been assumed as an estimator of the error and its general expression, valid for the most common sampling strategies used in practice, has been deduced. This asymptotic error is a function of the frequency response of the digital filter used and, eventually, of the characteristic function of the probability distribution selected for the random variables generating the sampling instants. Finally, the particular formulae for different sampling strategies and filtering algorithms are discussed and compared.

\section{Introduction}

This research aimed to develop a procedure for the comparison of the effects of the different sampling strategies (synchronous, asynchronous, equally spaced and random) and filtering algorithms on the output digital instruments which estimate the time average of a signal processed with a nonlinear conversion of multiple inputs (e.g. wattmeters, RMS voltmeters, ...). Once the DC quantity to be estimated was defined and identified as the true value [21], the output signal was expressed as a function of the true value, through the influences of the different sampling and filtering techniques (see Section 2). The measurement error, i.e. the difference between the

Paper 8810A (E12), first received 4th March and in revised form 20th December 1991

M.A. Catelani and G. Iuculano are with the Facoltà di Ingegneria, via S. Marta 3, 50139 Firenze, Italy

D. Miri, F. Filicori and A. Menchetti are with the Facoltà di Ingegneria, viale Risorgimento 2, 40136, Bologna, Italy

IEE PROCEEDINGS-A, Vol. 139, No. 4, JULY 1992 output signal and the true value, depends on some incidental nuisance parameters which locally restrict its evaluation to the choice of particular values of these parameters. To overcome this difficulty, according to the Bayesian approach, we consider the nuisance parameters as random variables with a distribution function correlated to the prior information available. This prior information can therefore be incorporated within the output signal, transforming it into a statistic that estimates the true value, no matter whether we have a single observation, we are in a particular reading occasion, or whether we are using a specified instrument based on the technique we are judging. The problem is led to evaluate the efficiency of an estimator of the true value, and this efficiency can be measured by introducing the mean-squared error. However, the intrinsic nuisance of the incidental parameters only disappears in every case if we evaluate the mean-squared error when the ranges in which they are estimated tend to be infinitely large, and so the asymptotic mean-squared error is assumed as a measure of the efficiency of the different sampling strategies and filtering techniques (see Section 3).

For the different techniques we are concerned with, the asymptotic mean-squared error coincides with the asymptotic variance which can be expressed as a sum of terms, each one of which is obtained by weighting the contribution relative to each spectral component of the converted signal. The weighting coefficients for a particular converted signal are easily deduced from a continuous weighting function once it has been specified whether the sampling strategy is asynchronous or synchronous. This weighting function can be expressed through the frequency response of the used digital filter and, for the random strategies, the common characteristic function of the random variables which generate the sampling instants (see Section 4).

For the different sampling strategies, we also assessed the possibility of utilising this weighting function as a quantity aimed at characterising the uncertainty within which the true value of the measure is estimated to have a specified probability (see Sections 5 and 7 ).

\section{Basic structure of sampling instrument based on nonlinear digital processing}

Fig. 1 shows the block diagram for a digital instrument which is based on a memoryless nonlinear conversion of 
the input signals and whose output is an estimate of the mean value of the converted signal. The nonlinear conversion can be effected normally, in a digital environ-

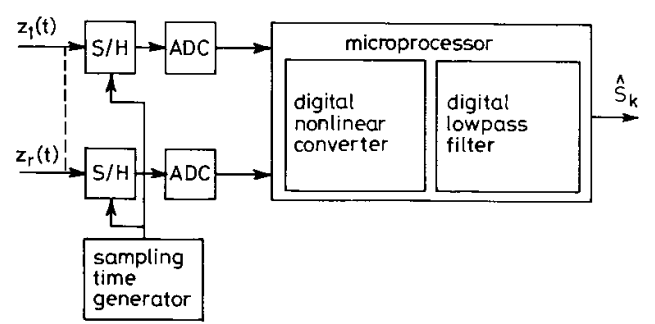

Fig. 1 Block diagram of all-digital instrument that measures time average of signal processed with nonlinear conversion of multiple inputs $\left(z_{1}, \ldots, z_{\text {, }}\right)$

ment, with a negligible error with respect to the errors introduced by the other circuits; one of the main problems, in terms both of accuracy and bandwidth is, instead, the choice of the sampling strategy and of the filtering algorithm. In the hypothesis that the nonlinear converter is memoryless and that the $\mathbf{S} / \mathrm{H}$ and ADC circuits can be considered ideal, we can exchange the nonlinear process with the sampling procedure, from a functional point of view; in this hypothesis the sampling procedure is directly effected on the converted signal (see Fig. 2a). Because the first block of Fig. $2 a$ (the nonlinear converter) does not influence the uncertainty and the bandwidth of the instrument, to study the errors arising from the sampling and filtering procedures we can assume the converted signal $s(t)$ as the input signal (see Fig. 2b).
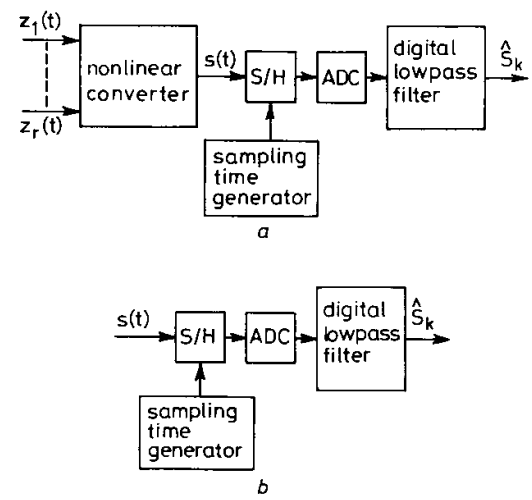

Fig. 2 Functional block diagrams used for performance analysis of instrument of Fig. 1

Many measurement instruments can be described by the block diagram of Fig. 1: the AC/DC converters of the memoryless type (the true RMS and the average responding voltmeters), the wattmeter, the vector voltmeter and the waveform analyser. For the last two instruments a suitable reference sinusoidal signal is required at one of the inputs. The block diagram of Fig. $2 b$ also represents a sampling DC voltmeter; our study, therefore, also allows us to evaluate the rejection to normal mode, i.e. superimposed noise signals.

In general we can assume that the converted signal $s(t)$, which is a function of the input signals $z_{1}(t), \ldots, z_{r}(t)$ in Fig. $2 a$, has a discrete spectrum and can be expressed by a generalised Fourier series in the form

$$
\begin{aligned}
s(t) & =g\left[z_{1}(t), \ldots, z_{r}(t)\right] \\
& =S_{0}+\sum_{\substack{q=-\infty \\
q \neq 0}}^{+\infty} A_{q} \exp \left(j \omega_{q} t\right)
\end{aligned}
$$

where $\omega_{-q}=-\omega_{q}, A_{-q}=A_{q}^{*}$ coincides with the complex conjugate of $A_{q}$ and the different $\omega_{q}$ do not necessarily have a common submultiple; $S_{0}$ represents the DC quantity to be measured which, in the time domain, is defined by

$$
S_{0}=\lim _{T_{0} \rightarrow \infty} \frac{1}{T_{0}} \int_{-T_{0} / 2}^{+T_{0} / 2} s(t) d t
$$

which we identify as the true value of the misurand [21]. When the converted signal is periodic with period $T_{1}=$ $2 \pi / \omega_{1}$ it results in $\omega_{q}=q \omega_{1}$, and the DC quantity $S_{0}$ can be calculated without considering, in eqn. 2 , the limit if $T_{o}$ coincides with the period of the signal $T_{o}=T_{1}$.

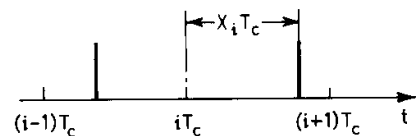

Fig. 3 Two successive sampling instants for random sampling technique defined by eqn. 3

Most of the sampling techniques that are used in practice can be defined by the following additive model (see Fig. 3):

$$
t_{i}=\left(i+X_{i}\right) T_{c}+\tau
$$

where, by writing any random variable in boldface letter, any sampling instant $t_{i}$ is given by the sum of a periodic component $i T_{c}$ and a random one $X_{i} T_{c} . T_{c}$ is a constant that usually takes the smallest possible value which is compatible with the maximum data acquisition and processing rate of the digital hardware adopted; $\boldsymbol{X}_{i}$ is the $i$ th of a set of random independent variables having a continuous distribution in the interval $-b$ to $+b$ with $0 \leqslant b<1$ (assuming $b=1 / 2$, the successive sampling instants are distributed in consecutive nonoverlapping intervals equal to $T_{c}$ ); the constant $\tau$ is the initial shift between the origin of the sequence of the instants, generated by the sampling time generator, and that to which the time domain of the signal $s(t)$ refers. Eqn. 3 defines a random sampling strategy and includes the synchronous and the asynchronous equally spaced sampling techniques (when $\boldsymbol{X}_{i}=0$ for any $i$ ). In the case of the asynchronous sampling techniques $T_{c}$ is not correlated with any spectral component of the signal $s(t)$ as the operation of the sampling time generator is independent from the input signals $z_{1}(t), \ldots, z_{r}(t)$. In the synchronous case (which can be used only for periodic signals) $T_{c}$ and $\tau$ are, instead, correlated with the input signals because the synchronised sampling time generator is controlled by one of these input signals; in such conditions $r$ will assume a value which depends not only on the synchronising input signal waveform, but also on the particular structure of the synchronising circuit adopted. In any case, the constant $T_{c}$ can be interpreted as the mean-sampling period, i.e. the mean value of the interval between two successive sampling instants.

The output of the $\mathrm{S} / \mathrm{H}$ circuit of Fig. $2 b$ is a discrete time signal $s\left(t_{i}\right)$, with $t_{i}$ defined by eqn. 3 ; therefore it can be represented mathematically as a sequence $\left\{s_{i}=s\left(t_{i}\right)\right\}$, $i=0, \pm 1, \ldots$. A common nonrecursive technique for appropriately smoothing this sequence is to introduce a 
discrete linear time invariant (LTI) system characterised by a finite impulse response (FIR filter) with constant values $a_{i}[1-3]$; by indicating with $\left\{\boldsymbol{S}_{k}\right\}, k=0, \pm 1, \ldots$, the sequence that represents the discrete time output signal of the instrument, we can write

$$
\hat{S}_{k}=\sum_{i=0}^{N-1} a_{i} s_{k-i}
$$

where the coefficients $a_{i}$ must be conveniently selected to achieve the prescribed filter characteristics. The time interval in which the $N$ samples of the input signals are taken to estimate the mean value of the converted signal $s(t)$, i.e. the response time of the instrument, is therefore $N T_{c}$. Obviously the time interval between two successive outputs of the instrument is independent from this response time because the outputs can be obtained partially by utilising the same samples.

The sequence of the sampling instants, which characterises each sampling strategy, is defined by eqn. 3. By substituting eqns. 1 and 3 into eqn. 4 we obtain

$$
\begin{aligned}
\hat{S}_{k}\left(\tau, \bar{X}_{k}\right)= & =\sum_{i=0}^{N-1} a_{i} S_{0}+\sum_{\substack{q=-\infty \\
q \neq 0}}^{+\infty} A_{q} \exp \left(j \omega_{q}\left[k T_{c}+\tau\right]\right) \\
& \times \sum_{i=0}^{N-1} a_{i} \exp \left(-j \omega_{q} i T_{c}\right) \exp \left(j \omega_{q} X_{k-i} T_{c}\right)
\end{aligned}
$$

where we have emphasised that the output sequence $\hat{S}_{k}$ is a function of the initial shift $\tau$ and of the vector of the successive $N$ sampling random variables $\bar{X}_{k}=\left\{X_{k}, X_{k-1}\right.$, $\left.\ldots, \boldsymbol{X}_{k-N+1}\right\}$. To avoid a distortion in the evaluation of the DC component of the converted signal $s(t)$, it is necessary to impose a scale factor equal to one

$$
\sum_{i=0}^{N-1} a_{i}=1
$$

\section{Criterion for performance analysis}

The output quantity $\hat{S}_{k}\left(\tau, \bar{X}_{k}\right)$, defined by eqn. 4 , is an estimate of the DC quantity $S_{0}$ and is affected by an error $e_{k}\left(\tau, \bar{X}_{k}\right)=\hat{S}_{k}\left(\tau, \bar{X}_{k}\right)-S_{0}$. This error, according to eqn. 5 , depends on the index $k$, which marks any output state of the instrument, on the initial shift $\tau$, and on the vector $\bar{X}_{k}$ of the successive $N$ sampling random variables. The incidental quantities $k$ and $\tau$ which appear in eqn. 5 occur as nuisance parameters because they locally condition the evaluation of the error by restricting it to the choice of a particular couple of values of $k$ and $\tau$. To eliminate the influence of these nuisance parameters, by foliowing the Bayesian approach [5] we can regard any couple of possible values $(k, \tau)$ as a realisation of a bivariate random variable $(k, \tau)$, with a priori probability distribution which must be selected by their intrinsic relationship to the prior information available. As far as $\boldsymbol{k}$ is concerned, it should be considered that every output state of the instrument has an equal chance of being selected; therefore, the innumerable sets of labels which mark the sequence of the output states of the instrument can be treated as a discrete random variable, uniformly distributed within the whole time interval in which these states are available. As far as $\tau$ is concerned, we must distinguish between the synchronous sampling techniques and the asynchronous ones. In the asynchronous cases, the sampling time generator (see Fig. 1b) is not controlled by any one of the input signals, so that any realisation of $\tau$ is independent of the converted signal $s(t)$ and, as it is strictly related to the turnon instant of the instrument, it belongs to a continuous set of possible equiprobable values distributed within any time interval. In the syn- chronous sampling technique, instead, $\tau$ depends on the synchronising circuit which generates the sequence of the sampling instants $t_{j}$ as a function of one of the input signals; consequently, $\tau$ is a function of the synchronising circuit used and the converted signal $s(t)$. If we want to take into account all the possible states of $\tau$, we should have to foresee all the possible synchronising operations for the same unknown signal and establish the possible functional relations which connect $\tau$ to the signal itself. Obviously, this is practically utopian and we propose to simulate an equivalent configuration in which $\tau$ may be assumed as a realisation of a continuous set of values distributed within some time interval $T$. To sum up, in both techniques the a priori ignorance concerning the nature of the parameter $\tau$ can be overcome by postulating a uniform distribution in a generic time interval $T$.

Consequently the error can be rewritten as

$$
e_{k}\left(\tau, \bar{X}_{k}\right)=\hat{S}_{k}\left(\tau, \bar{X}_{k}\right)-S_{0}
$$

where $\hat{S}_{k}\left(\tau, \bar{X}_{k}\right)$ is a combined function of $k, \tau$ and $\bar{X}_{k} ; \boldsymbol{k}$ is a discrete random variable, in general with $2 h+1$ values, uniformly distributed and symmetric with respect to a conventional origin, $\tau$ is a continuous random variable uniformly distributed within a prefixed interval centred round another conventional origin, and $\overline{\boldsymbol{X}}_{\boldsymbol{k}}$ is the random vector whose components are the random variables, introduced in eqn. 3 , generated in the particular measurement operation. With eqn. 7 we have incorporated into the local error the prior distribution available for the incidental parameters transforming the output signal into a static which estimates the true value $S_{o}$. Now the problem is reduced to measure the efficiency of an estimator of the true value $S_{o}$. This estimator is assumed as inversely proportional to the mean-squared error, i.e. the expected value of the squared error, defined by [4]

$$
\left\langle e_{k}^{2}\left(\tau, \bar{X}_{k}\right)\right\rangle=\frac{1}{2 h+1} \sum_{k=-h}^{+h} \frac{1}{T} \int_{-T / 2}^{+T / 2}\left\langle e_{k}^{2}\left(\tau, \bar{X}_{k}\right)\right\rangle d \tau
$$

where the symbol $\langle\cdot\rangle$ indicates the statistical mean value and, for the sake of simplicity, we have considered the conventional time origin for the initial shift $\tau$ equal to zero. To incorporate all the possible $a$ priori chances and avoid the influence of the conventional time origin on the instrument performance, the number of output states, i.e $2 h+1$, and the excursion of the initial shift $\tau$, i.e. $T$, must be sufficiently large, and theoretically they must tend to infinite. Therefore, a useful criterion for comparing the relative efficiencies of the different measurement processes is to consider the asymptotic mean-squared error

$$
E^{2}=\lim _{\substack{h \rightarrow \infty \\ T \rightarrow \infty}}\left\langle e_{k}^{2}\left(\tau, \bar{X}_{k}\right)\right\rangle=\Delta S_{0}^{2}+\sigma^{2}
$$

where, by introducing the asymptotic expectation

$$
\bar{S}=\lim _{\substack{h \rightarrow \infty \\ T \rightarrow \infty}}\left\langle\hat{S}_{k}\left(\tau, \bar{X}_{k}\right)\right\rangle
$$

$\Delta S_{0}$ represents the asymptotic bias

$$
\Delta S_{0}=\bar{S}-S_{0}
$$

whereas

$$
\begin{aligned}
\sigma^{2} & =\lim _{\substack{h \rightarrow \infty \\
T \rightarrow \infty}}\left\langle\left[\hat{S}_{k}\left(\tau, \bar{X}_{k}\right)-\left\langle\hat{S}_{k}\left(\tau, \bar{X}_{k}\right)\right\rangle\right]^{2}\right\rangle \\
& =\lim _{\substack{h \rightarrow \infty \\
T \rightarrow \infty}}\left\langle\hat{S}_{k}^{2}\left(\tau, \bar{X}_{k}\right)\right\rangle-\bar{S}^{2}
\end{aligned}
$$

defines the asymptotic variance. 
The asymptotic mean-square error characterises the range within which the true value $S_{o}$ can be estimated with a specified confidence coefficient $\left(1-1 / \eta^{2}\right)$ through the following Chebychev inequality

$$
\lim _{\substack{h \rightarrow \infty \\ T \rightarrow \infty}} P\left\{\hat{S}_{k}\left(\tau, \bar{X}_{k}\right)=S_{0} \pm \eta E\right\} \geqslant 1-\frac{1}{\eta^{2}}
$$

\section{Weighting function}

It can be shown (Appendix 10.1) that, for every sampling procedure (synchronous or asynchronous) defined by eqn. 3 and for every filtering algorithm defined by eqn. 4 , the asymptotic mean-squared error, in the hypothesis of a scale factor equal to one (see eqn. 6), coincides with the asymptotic variance $\sigma^{2}$; besides, this asymptotic variance $\sigma^{2}$ can be calculated by the equation (see eqn. 68 in Appendix 10.1)

$$
\sigma^{2}=2 \sum_{q=1}^{\infty}\left|A_{q}\right|^{2} W^{2}\left(f_{q} T_{c}\right)
$$

This equation shows that the asymptotic variance can be expressed as a series, each of whose term is given by the square of the RMS value of each converted-signal spectral component multiplied by a weighting coefficient. The infinite sequence of the weighting coefficients $W^{2}\left(f_{1} T_{c}\right)$, $W^{2}\left(f_{2} T_{c}\right), \ldots$ can be derived from a continuous weighting function $W^{2}\left(f T_{c}\right)$ by determining its values at the successive occurrence points $f_{1} T_{c}, f_{2} T_{c}, \ldots$, i.e. the products between each signal spectral frequency and the meansampling period $T_{c}$.

In the following the expression of the weighting function is deduced by considering only the errors which arise from the sampling and the filtering procedures; the other causes of error, e.g. the bandwidth limitations introduced by the sample and hold circuits, must be accounted separately. In this hypothesis, the behaviour of the weighting function $W^{2}\left(f T_{c}\right)$ as a function of the normalised frequency $f T_{c}$ and the possible occurrence points at which the weighting coefficients are evaluated describe completely the performance of the instrument.

By denoting with $\Phi\left(\omega T_{c}\right)$ the characteristic function [4], in the normalised frequency (rad) domain $\omega T_{c}$, of the random variable $\boldsymbol{X}_{j}$ which appears in eqn. 3

$$
\Phi\left(\omega T_{c}\right)=\left\langle\exp \left(j \omega T_{c} X_{j}\right)\right\rangle
$$

and with $H\left(\omega T_{c}\right)$ the normalised frequency response of the digital filter $[1-3]$ defined by eqn. 4

$$
H\left(\omega T_{c}\right)=\sum_{i=0}^{N-1} a_{i} \exp \left(-j \omega i T_{c}\right)
$$

the weighting function $W^{2}\left(f T_{c}\right)$ for the different sampling techniques considered here can be deduced from eqn. 69 in Appendix 10.1 by replacing the discrete variable $f_{4}$ by a continuous one $f$

$$
\begin{aligned}
W^{2}\left(f T_{c}\right)= & W_{R}^{2}\left(f T_{c}\right) \\
= & \sum_{i=0}^{N-1} a_{i}^{2}+\left|\Phi\left(2 \pi f T_{c}\right)\right|^{2} \\
& \times\left(\left|H\left(2 \pi f T_{c}\right)\right|^{2}-\sum_{i=0}^{N-1} a_{i}^{2}\right)
\end{aligned}
$$

the index $R$ is introduced to emphasise the contributions of the random variables to the sampling technique. When the sampling technique is of an equally spaced type $\left(X_{j}=0\right.$ in eqn. 3), the characteristic function $\Phi\left(\omega T_{c}\right)$ becomes equal to one; therefore, from eqn. 17 it can be deduced that the weighting function coincides with the square of the magnitude of the frequency response of the FIR filter utilised

$$
W^{2}\left(f T_{c}\right)=W_{E}^{2}\left(f T_{c}\right)=\left|H\left(2 \pi f T_{c}\right)\right|^{2}
$$

where the index $E$ means that the sampling strategy is of an equally spaced type, i.e. without any random contribution.

In the following Sections the weighting functions $W\left(f T_{c}\right)$ using different FIR filters are determined. To distinguish these different functions we shall introduce in $W\left(f T_{c}\right)$ a second index, relative to the window used for designing the FIR filter. To determine the occurrence points of the weighting coefficients it is necessary to specify whether the sampling technique is asynchronous or synchronous.

\section{Performance analysis for the equally spaced} asynchronous sampling technique

In the asynchronous case the initial shift $\tau$ which appears in eqn. 3 is strictly related to the turnon instant of the instrument, the instant that can be regarded as the realisation of a random variable; therefore, the asymptotic mean-squared error defined by eqn. 9 allows us to not only compare the different sampling strategies and filtering algorithms, but also to determine the uncertainty of each instrument based on an asynchronous sampling strategy. Moreover, as $T_{c}$ is a constant not correlated with any spectral component of the converted signal, the product $f_{a} T_{c}$ can assume any positive real value.

To evaluate the output signal of the instrument we can average the last $N$ signal samples $\left(a_{i}=1 / N\right.$ in eqn. 4), i.e. utilise an $N$-point rectangular window to realise the FIR filter. In this hypothesis, for the property of the geometric progression, the frequency response of this filter (see eqn. 16) results in

$$
\begin{aligned}
H_{R}\left(f T_{c}\right) & =\sum_{i=0}^{N-1} \exp \left(-j \omega i T_{c}\right) \\
& =\frac{1-\exp \left(-j \omega N T_{c}\right)}{1-\exp \left(-j \omega T_{c}\right)} \\
& =\exp \left(j \pi(N-1) f T_{c}\right) \frac{\sin \left(\pi f N T_{c}\right)}{\sin \left(\pi f T_{c}\right)}
\end{aligned}
$$

where we have introduced the index $R$ to indicate that the window is of a rectangular type. By substituting this equation into eqn. 18 and by introducing a second index $R$ in the weighting function to specify the window, we obtain [17]

$$
W_{E R}^{2}\left(f T_{c}\right)=\frac{1}{N^{2}} \frac{\sin ^{2}\left(N \pi f T_{c}\right)}{\sin ^{2}\left(\pi f T_{c}\right)}=\frac{\operatorname{sinc}^{2}\left(N f T_{c}\right)}{\operatorname{sinc}^{2}\left(f T_{c}\right)}
$$

The weighting function $W_{E R}^{2}\left(f T_{c}\right)$ is periodic with respect to $f T_{c}$ with a period equal to one and assumes, at the extreme of each period, its absolute maxima that are equal to one, independently of any prefixed value of $N$. By referring to the first period, $W_{E R}^{2}\left(f T_{c}\right)$ is symmetric with respect to $1 / 2$; besides, it assumes $(N-1)$ zeros for

$$
f_{i \min } T_{c}=\frac{i}{N}
$$

with $i=1, \ldots, N-1$, and $(N-2)$ maxima for (see Appendix 10.2)

$$
f_{i \max } T_{c}=f_{i \min } T_{c}+\frac{1}{2 N}=\frac{1}{N}\left(i+\frac{1}{2}\right)
$$


with $i=1, \ldots, N-2$. The values of this maxima are given by

$$
W_{E R}^{2}\left(f_{i \max } T_{c}\right)=\frac{1}{N^{2}} \frac{1}{\sin ^{2}\left(\frac{(2 i+1)}{N} \frac{\pi}{2}\right)}
$$

Fig. 4 shows the plot of $W_{E R}^{2}$ for two successive periods and $N=7$; this very low value of $N$ has been chosen only to show the whole shape of the function in every period. By assuming, instead, $N=10^{4}$ the minimum value assumed by the maxima expressed by eqn. 23 is equal to $10^{-8}$; therefore a unit amplitude harmonic component of the converted signal at this frequency gives a contribution to the asymptotic variance equal to $0.5 \times 10^{-8}$ (see eqn. 14). In the hypothesis that this is the unique harmonic component of the converted signal, the standard deviation of the output signal of the instrument results equal to $0.7 \times 10^{-4}$ (see eqn. 14).

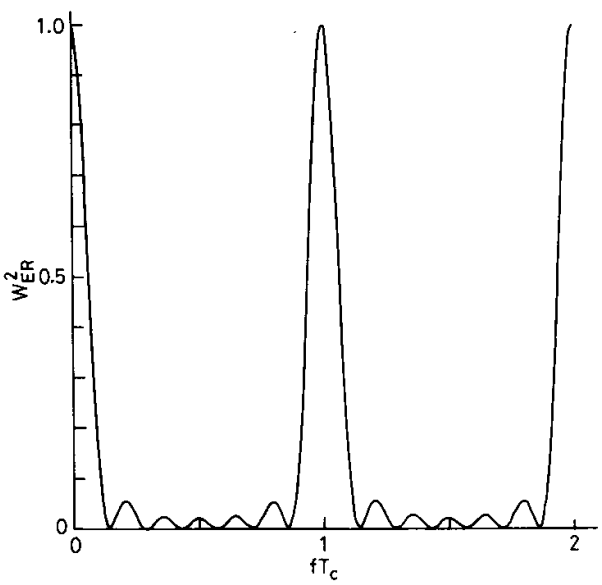

Fig. 4 Weighting function $W_{E R}^{2}$, for equally spaced sampling technique and rectangular window $N=7$, as a function of the normalised frequency $f T_{c}$

From eqn. 23 it is possible to deduce the frequency range in which the maxima of $W_{E R}^{2}\left(f T_{c}\right)$ assume a value that is not higher than a prefixed value, i.e. the bandwidth in which the contribution of every harmonic of the converted signal to the variance in the hypothesis of an asynchronous sampling technique is not higher than a prefixed value; this bandwidth, in any case, will certainly be smaller than $f_{c}$.

To attenuate the contributions of the alternate components of the converted signal $s(t)$ more efficiently it is necessary to increase the value of $N$ and/or to modify the filter, e.g. by using a trapezoidal window with clipped ends in which the coefficients $a_{i}$ of eqn. 4 are defined according to the following conditions:

$$
a_{i}= \begin{cases}(i+1) / m n & \text { for } 0 \leqslant i \leqslant n-1 \\ n-m n & \text { for } n \leqslant i \leqslant m-2 \\ (m+n-i-1) / n m & \text { for } m-1 \leqslant i \leqslant n+m-2\end{cases}
$$

where $m>n$ and $N=n+m-1$ have been imposed (see Fig. 5). For $m=n$ the window becomes triangular with clipped ends, for $n=1$ and $m>1$ it becomes rectangular. The $a_{i}$ coefficients given by eqn. 24 satisfy eqn. 6 and result in

$$
\begin{aligned}
\sum_{i=0}^{n+m-2} a_{i} & =\sum_{i=0}^{n-1} \frac{i+1}{m n}+\sum_{i=n}^{m-2} \frac{1}{m}+\sum_{i=m-1}^{m+n-2} \frac{m+n-i-1}{m n} \\
& =\sum_{i=0}^{n-1} \frac{i+1}{m n}+\sum_{i=n}^{m-2} \frac{1}{m}+\sum_{i=0}^{n-1} \frac{n-i}{m n}=1
\end{aligned}
$$

Another important property is the following

$$
\begin{aligned}
\sum_{i=0}^{n+m-2} a_{i}^{2}= & \frac{1}{(n m)^{2}}\left[\sum_{i=0}^{n-1}(1+i)^{2}+\sum_{i=n}^{m-2} n^{2}\right. \\
& \left.+\sum_{i=m-1}^{m+n-2}(m+n-i-1)^{2}\right] \\
= & \frac{1}{(n m)^{2}}\left[2 \sum_{i=1}^{n} i^{2}+n^{2}(m-n-1)\right] \\
= & \frac{1}{(n m)^{2}}\left[\frac{n(n+1)(2 n+1)}{3}+n^{2}(m-n-1)\right]
\end{aligned}
$$

This last result coincides with the formula (48) calculated in a previous paper [16]. Obviously, for $m<n$, in all the previous results it is necessary to exchange the contribution between $m$ and $n$.

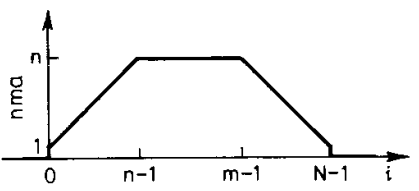

Fig. 5 Trapezoidal window with clipped ends for FIR filter $N=n+m-1 ; m>n$

It can be shown that, for the trapezoidal window with clipped ends, the frequency response of the filter is defined by the following expression (see Appendix 10.3)

$$
\begin{aligned}
H\left(2 \pi f T_{c}\right) & =H_{T}\left(2 \pi f T_{c}\right) \\
& =e^{-j \pi(m+n-2) f T_{c}} \frac{\operatorname{sinc}\left(n f T_{c}\right) \operatorname{sinc}\left(m f T_{c}\right)}{\operatorname{sinc}^{2}\left(f T_{c}\right)}
\end{aligned}
$$

where the index $T$ means trapezoidal window with clipped ends. By substituting this equation in eqn. 18 we obtain the expression of the weighting function $W_{E T}^{2}$ (the first index $E$ means equally spaced sampling and the second index $T$ means trapezoidal window with clipped ends) for this case $[17]$

$$
W^{2}\left(f T_{c}\right)=W_{E T}^{2}\left(f T_{c}\right)=\frac{\operatorname{sinc}^{2}\left(n f T_{c}\right) \operatorname{sinc}^{2}\left(m f T_{c}\right)}{\operatorname{sinc}^{4}\left(f T_{c}\right)}
$$

Recalling that $N=n+m-1$, it is easy to verify that eqn. 28 contains the following particular cases

(a) triangular window with clipped ends for $n=m=(N+1) / 2$, i.e.

$$
W^{2}\left(f T_{c}\right)=W_{E B}^{2}\left(f T_{c}\right)=\frac{\operatorname{sinc}^{4}\left([(N+1) / 2] f T_{c}\right)}{\operatorname{sinc}^{4}\left(f T_{c}\right)}
$$

where we have used the second index $B$ because it reminds to the Bartlett window

(b) rectangular window for $n$, or $m$, equal to one (see eqn. 20).

The same result of eqn. 28 could be obtained with a double averaging operation that averages $m$ terms, each of which is the sum of successive $n$ samples of the converted signal. In fact, the frequency response of a filter having a trapezoidal window with clipped ends can be expressed as a product of two frequency responses, one 
relative to a filter which averages $n$ values and the other $m$ values (see Appendix 10.3 and Reference 1)

$$
H_{T}\left(2 \pi f T_{c}\right)=H_{R n}\left(2 \pi f T_{c}\right) H_{R m}\left(2 \pi f T_{c}\right)
$$

where the second index specifies the values averaged in each rectangular window. By substituting eqn. 18 into this equation, the weighting function of a trapezoidal window with clipped ends can be expressed as a product of two weighting functions, one relative to a filter which averages $n$ values and the other $m$ values

$$
W_{E T}^{2}\left(f T_{c}\right)=W_{E R n}^{2}\left(f T_{c}\right) W_{E R m}^{2}\left(f T_{c}\right)
$$

Also the weighting function $W_{E T}^{2}\left(f T_{c}\right)$ is periodic with respect to $f T_{c}$ with a period equal to one, and is symmetric with respect to each integer value, where it assumes its absolute maximum, equal to one. It can be shown that, by imposing a prefixed number $N$ of sampling instants, eqn. 28 , considered as a function only of $n$ or $m$, joins minima values when $n=m=(N+1) / 2$, excluding the points where it assumes values equal to one. For example, Fig. $6 a$ shows the weighting function
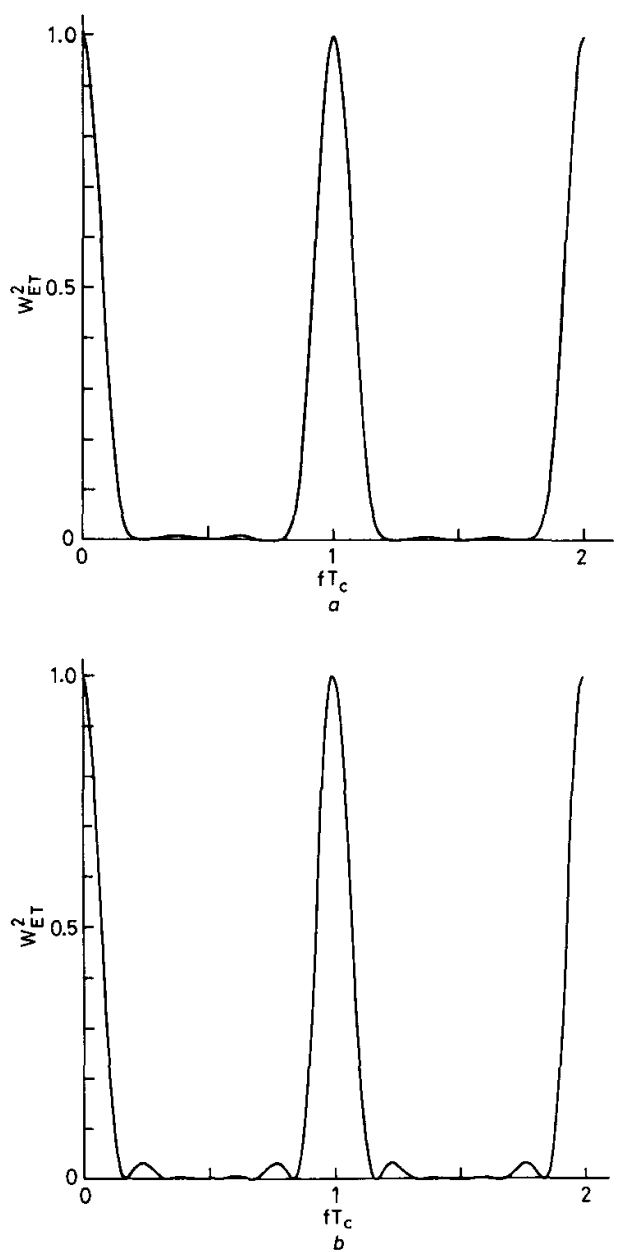

Fig. 6 Weighting function for equally spaced sampling technique a Triangular window with clipped ends

$N=7: n=m=4$

$b$ Trapezoidal window with elipped ends
$N=7: n=2 ; m=6$

146 for a triangular window with $n=m=4$, and Fig. $6 b$ shows a trapezoidal window with $n=2$ and $m=6$, for the same number $N=7$. In the hypothesis that $n=m=(N+1) / 2$ (triangular window with clipped ends), the frequencies where the weighting function assumes null or maxima values are the same as those deduced for the weighting function with a rectangular window (see eqns. 21 and 22); i.e. eqn. 31 , when $m=n$, becomes the square of the rectangular weighting function $W_{E R_{n}}^{2}$. Consequently the values of these maxima in the first period can be deduced, taking into account eqns. 23 and 31 , from the following expression

$$
W_{E B}^{2}\left(f_{i \max } T_{c}\right)=\frac{16}{(N+1)^{4}} \frac{1}{\sin ^{4}\left(\frac{(2 i+1) \pi}{N+1}\right)}
$$

These maxima must be compared with the maxima of a rectangular window (see eqn. 23) for the same number $N$ of sampling instants used to obtain each output of the instrument. Recalling that the value of $N$ is much greater than one, a considerable improvement in the performance of the instrument can be obtained with a triangular window with clipped ends. For example, by supposing $N=10^{4}$, the minimum value assumed by the maxima expressed by eqn. 32 is equal to $1.6 \times 10^{-15}$.

\section{Performance analysis for random asynchronous sampling technique}

By supposing the random variable $\boldsymbol{X}_{j}$ of eqn. 3 uniformly distributed in the interval $-b$ to $+b$, with $0<b<1$ (see Fig. 7), the characteristic function becomes

$$
\begin{aligned}
\Phi\left(\omega T_{c}\right) & =\left\langle\exp \left(j \omega T_{c} X_{j}\right\rangle\right. \\
& =\frac{\sin \left(b \omega T_{c}\right)}{\left.b \omega T_{c}\right)} \\
& =\operatorname{sinc}\left(2 b f T_{c}\right)
\end{aligned}
$$

Besides, by using an $N$-point rectangular window to realise the FIR filter and by recalling eqn. 18, eqn. 17 can be rewritten as follows:

$$
\begin{aligned}
W^{2}\left(f T_{c}\right) & =W_{R R}^{2}\left(f T_{c}\right) \\
& =\frac{1}{N}+\operatorname{sinc}^{2}\left(2 b f T_{c}\right)\left[W_{E R}^{2}\left(f T_{c}\right)-\frac{1}{N}\right]
\end{aligned}
$$

where $W_{E R}^{2}\left(f T_{c}\right)$ is expressed by eqn. 20. For $f=0$ we obtain $W_{R R}^{2}(0)=W_{E R}^{2}(0)=1$. For $0<f T_{c} \ll 1 / 2$, $\operatorname{sinc}^{2}\left(2 b f T_{c}\right)$ can be considered approximately equal to one independently of $b$; in this frequency range $W_{R R}^{2}\left(f T_{c}\right)$ can therefore be approximated by $W_{E R}^{2}\left(f T_{c}\right)$. For $f_{s} T_{c}<$ $f T_{c}<1, W_{R R}^{2}\left(f T_{c}\right)$ increases, as shown in Fig. 8. When $f T_{c} \geqslant 1$, the shape of $W_{R R}^{2}\left(f T_{c}\right)$ depends significantly on the given value of parameter $b$. For instance, by selecting $b=1 / 2$ (i.e. when the random sampling instant $t_{j}$ in eqn. 3 is distributed within an interval equal to the time con-

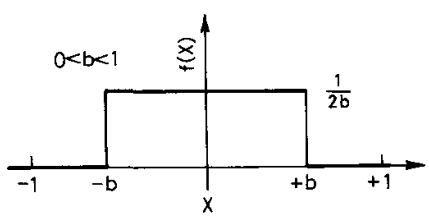

Fig. 7 Common probability density for sequence of random variables $X_{k}, X_{k-1}, \ldots, X_{k-N+1}$ in hypothesis of uniform distribution within interval
$(-b,+b)$

IEE PROCEEDINGS-A, Vol. 139, No. 4, JULY 1992 
stant $T_{c}$ ) we obtain $[16]$

$$
W_{\mathrm{RR}}^{2}\left(f T_{c}\right)=\frac{1}{N}+\operatorname{sinc}^{2}\left(f T_{c}\right)\left[W_{E R}^{2}\left(f T_{c}\right)-\frac{1}{N}\right]
$$

therefore the result is

$$
W_{R R}^{2}\left(f T_{c}\right) \simeq \frac{1}{N} \text { for } f T_{c} \geqslant 1
$$

In fact, $\operatorname{sinc}^{2}\left(f T_{c}\right)$ becomes identically null for $f T_{c}=r$, with $r=1,2, \ldots$, while $W_{E R}^{2}(r)$ is equal to one; for every other frequency value the result is $\operatorname{sinc}^{2}\left(f T_{c}\right) \ll 1$ and the product $\operatorname{sinc}^{2}\left(f T_{c}\right) W_{E R}^{2}\left(f T_{c}\right)$ is nearly equal to zero. Obviously this sampling technique, being of an asynchronous type, from eqn. 36 we can therefore deduce that the bandwidth of this instrument is not limited by the average sampling frequency $1 / T_{c}$; it is however convenient to remember that, in this study, we have not
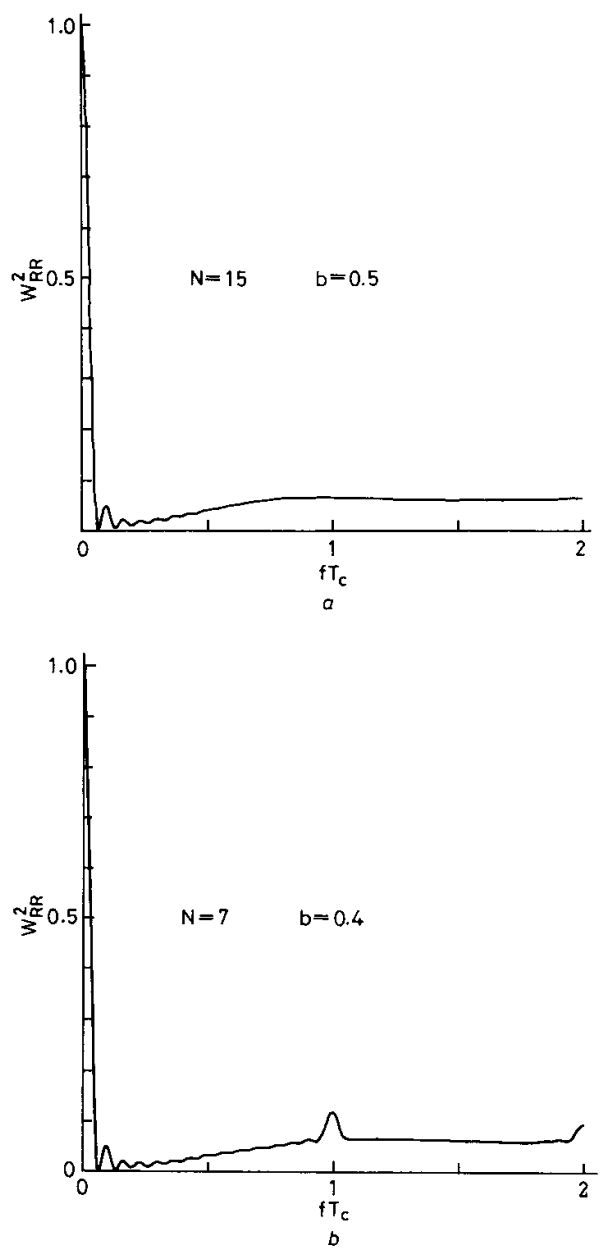

Fig. 8 Weighting function of random sampling technique defined by eqn. 3

Random variable is supposed as uniformly distributed in an interval of the mean sampling period $T_{\mathrm{c}}$, and the FIR filter is characterised by a rectangulat window. $a$ Interval equal to $T_{c}$
$N=15 ; b=0.5$

$b$ Interval slightly inferior to $T_{c}$

$N=7 ; b=0.4$

IEE PROCEEDINGS-A, Vol.139, No. 4, JULY 1992 taken into account the bandwidth limitations of the sample/hold circuits. Fig. $8 a$ shows the plot of the weighting function $W_{E R}^{2}\left(f T_{c}\right)$ in the hypothesis of $N=15$.

By means of this sampling technique a broadband instrument can be implemented easily; each channel requires a pair of $\mathrm{S} / \mathrm{H}$ devices and $\mathrm{ADC}$ (which operate alternately for odd and even sampling instants) because the time interval between the first and the last of a consecutive triplet of samples can never be smaller than $T_{c}$ [16]. Besides, it is necessary to verify that the errors introduced by the $\mathrm{S} / \mathrm{H}$ and $\mathrm{ADC}$ are negligible. To this end it was made the DC calibration of the ADC of a prototype by imposing that the difference between the output quantity of the instrument and the DC quantity common to all the inputs was negligible with respect to the standard deviation deduced from eqns. 14 and 35 ; then, by comparing the theoretical and the empirical values of the weighting function for the asynchronous equally spaced and the random one with $b=1 / 2$, it was found that they were in very good agreement [19]. This confirms the validity of the theoretical model proposed.

In practice the value of parameter $b$ cannot be exactly equal to $1 / 2$, i.e. the random variable $X_{i}$ of eqn. 3 is distributed within an interval slightly different from $(-1 / 2$, $+1 / 2$ ), owing to a non-ideal implementation of the random sampling strategy. When the parameter $b$ is different from $1 / 2$, from eqn. 34 it can be deduced that the weighting function $W_{R R}^{2}\left(f T_{c}\right)$ assumes sharp peaks (which depend on the value of $b$ ) for $f T_{c}=r$, with $r=1,2, \ldots$, as shown in Fig. $8 b$ where the parameter values $2 b=0.8$ and $N=7$ have been chosen. Assuming for the parameter $b$ a value slightly different from $1 / 2(b=1 / 2+\varepsilon$ with $\varepsilon$ conveniently small, for instance $|\varepsilon|<1 / 40$, i.e. $0.475<b<0.525$ ), the feasibility of the following approximation can be verified

$$
\operatorname{sinc}^{2}(2 b r)=\operatorname{sinc}^{2}(r+2 \varepsilon r) \simeq 4 \varepsilon^{2} \text { for } r=1,2, \ldots
$$

which is plotted in Fig. 9 as a function of $\varepsilon$. Therefore the weighting function for $f T_{c}=r$ with $r=1,2, \ldots$ assumes a maximum value given by $[20]$

$$
W_{R R}^{2}(r) \simeq \frac{1}{N}+4 \varepsilon^{2} \quad \text { for } r=1,2, \ldots
$$

From this equation it can be deduced that the two components which effect the value of these maxima are independent of each other; the first is a function of $N$, i.e. the number of samples which are taken to evaluate each output, the second, due to the sharp peaks, depends only on the difference between $b$ and $1 / 2$ (however, the width of these sharp peaks is approximately equal to $1 / N$ ). Therefore, if $N$ is sufficiently large, the inferior limit of the

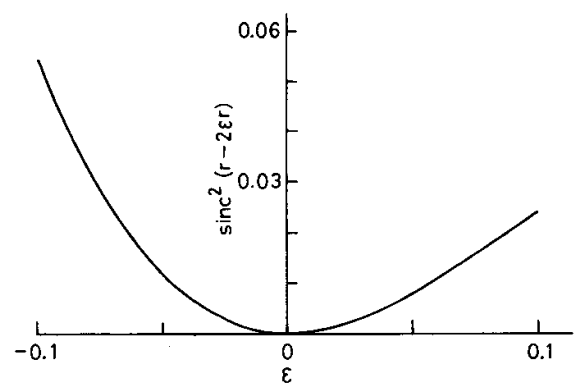

Fig. 9 Graph of eqn. 37 for $r=1$ as function of $\varepsilon$ 
weighting function is imposed by the uncertainty with which the condition $b=1 / 2$ is realised; this result is quite important from a practical point of view because it provides a quantitative criterion for determining how accurate the implementation of the random sampling strategy should be.

In the case of a trapezoidal window with clipped ends of the type shown in Fig. 5, the weighting function can be deduced by recalling eqn. 18 and by substituting eqns. 26 , 33 and 28 in eqn. 17

$$
W_{R T}^{2}\left(f T_{c}\right)=G+\operatorname{sinc}^{2}\left(2 b f T_{c}\right)\left[W_{E T}^{2}\left(f T_{c}\right)-G\right]
$$

where $G$ is given by eqn. 26

$$
\begin{aligned}
& G=\sum_{i=0}^{m+n-2} a_{i}^{2} \\
&=\frac{1}{(m n)^{2}}\left[\frac{n(n+1)(2 n+1)}{3}+n^{2}(m-n-1)\right] \\
& \quad \text { with } 1<n \leqslant m
\end{aligned}
$$

By comparing eqns. 34 and 39 it can be deduced that, for $0<f T_{c} \ll 1 / 2, \quad W_{R T}^{2}\left(f T_{c}\right)$ can be approximated by $W_{E T}^{2}\left(f T_{c}\right)$; instead, by imposing $b=1 / 2$ (i.e. the random sampling instant is distributed within a time interval equal to the time constant $T_{c}$ ), for $f T_{c} \geqslant 1$ this results in $W_{R T}^{2}\left(f T_{c}\right) \simeq G$. In the hypothesis that $n=m \simeq N / 2$ $(N \gg 1)$ we obtain $G \simeq 4 /(3 N)$, which is only slightly different from $1 / N$. Therefore, for $f T_{c} \geqslant 1$, the weighting functions for the rectangular (see eqn. 35) and the triangular windows with clipped ends practically coincide; this means that the triangular window with clipped ends is convenient in the asynchronous equally sampling technique, not in the random one [16].

7 Performance analysis for synchronous sampling techniques

The synchronous sampling methods can be adopted only when the input signals are strictly periodic with periods having a least-common multiple, denoted by $T_{1}$; therefore the converted signal $s(t)$ is strictly periodic with period $T_{1}=1 / f_{1}$ and can be expressed by the Fourier series derived from eqn. 1 with

$$
\omega_{q}=q \omega_{1}=q 2 \pi f_{1}
$$

being $q$ integer. In such conditions $S_{0}$ can be defined by eqn. 2 without resorting to the limit if $T_{o}$ coincides exactly with $T_{1}$.

In a synchronous sampling technique every sequence of the sampling instants used to generate an output signal of the instrument is synchronous with the converted signal and can be selected with equal or different initial shift $\tau$ (see eqn. 3) with respect to the converted signal.

The most common, synchronous sampling method is of an equally spaced type with a constant initial shif which depends on the synchronising circuit; therefore the output of the instrument is constant $[9,11]$ but, for the same value $S_{0}$ of the converted signal, it can be a function of the waveform of the synchronising input signal. The asymptotic mean-squared error defined by eqn. 9 represents a bound for the uncertainty of the instrument, because the range of the initial shift, for any prearranged synchronising circuit, is defined and contained in the period $T_{1}$ [9]; obviously, in the synchronous techniques, restoring to infinite is tantamount to joining the period
$T_{1}$. It is important to emphasise that the range of $\tau$ can be found when we select a prefixed synchronising circuit; in this hypothesis the uncertainty of the instrument can be evaluated by imposing that the excursion of $\tau$ for the evaluation of the mean-squared error coincides with that range. Instead, if the shift introduced by the synchronising circuit is fixed, it is a priori known and the uncertainty analysis is reduced to the evaluation of the difference between the output of the instrument and $S_{o}$.

Another sampling technique of an equally spaced type is that in which the initial shift $\tau$ is randomised [12], i.e. the randomisation of the initial shift is included in the sampling technique; in this case the output of the instrument is time varying and eqn. 9 also allows for the uncertainty estimation of the instrument.

In a synchronous sampling method of an equally spaced type the weighting function is expressed by eqn. 18. The synchronisation condition entails that the product $f_{1} T_{c}$ is fixed and independent, both of the fundamental frequency $f_{1}$ and the sampling period $T_{c}$. Consequently the weighting coefficients that appear in eqn. 14 are the values of the weighting function at the integer multiplies of the fixed value $f_{1} T_{c}$

By using an $N$ point rectangular window for the implementation of the FIR filter, the weighting function is expressed by eqn. 20; in the further hypothesis that a multiple integer of the sampling frequency $f_{\mathrm{c}}$ is equal to a multiple integer of the fundamental frequency $f_{1}$ [9]

$$
p f_{c}=u f_{1}
$$

where $p$ and $u$ are positive integers having no common submultiple, the weighting coefficient for the generic harmonic $q$ results in

$$
\begin{aligned}
W_{E R}^{2}\left(f_{q} T_{c}\right) & =W_{E R}^{2}\left(q f_{1} T_{c}\right) \\
& =W_{E R}^{2}\left(q \frac{p}{u}\right) \\
& =\frac{\operatorname{sinc}^{2}\left(N q \frac{p}{u}\right)}{\operatorname{sinc}^{2}\left(q \frac{p}{u}\right)}
\end{aligned}
$$

If we select the number $u$ of the samples in every $p T_{1}$ period of the converted signal equal to the length $N$ of the rectangular window, this results in

$$
\begin{aligned}
W_{E R}^{2}\left(q \frac{p}{N}\right) & =\frac{\operatorname{sinc}^{2}(q p)}{\operatorname{sinc}^{2}\left(q \frac{p}{N}\right)} \\
& =\left\{\begin{array}{ll}
1 & \text { for } q=z N \\
0 & \text { for } q \neq z N
\end{array} \text { with } z\right. \text { integer }
\end{aligned}
$$

being $q, p$ and $N$ integers. In Fig. 10 the weighting function and the values of the weighting coefficients are given in the hypothesis of $N=u=7$ and $p=2$. By introducing these coefficients in eqn. 14 , and by recalling that it results in $E^{2}=\sigma^{2}$ (see Section 3), the mean-squared error can be expressed as follows

$$
E^{2}=2 \sum_{z=1}^{+\infty}\left|A_{z N}\right|^{2}
$$

The contribution of the spectral component of the converted signal to the mean-squared error is nonzero only for any $q$ multiple integer of $u=N$. In other terms, the 
choice of a rectangular window of length $N=u$ entails that the asymptotic variance does not depend on the shape of the weighting function among the successive

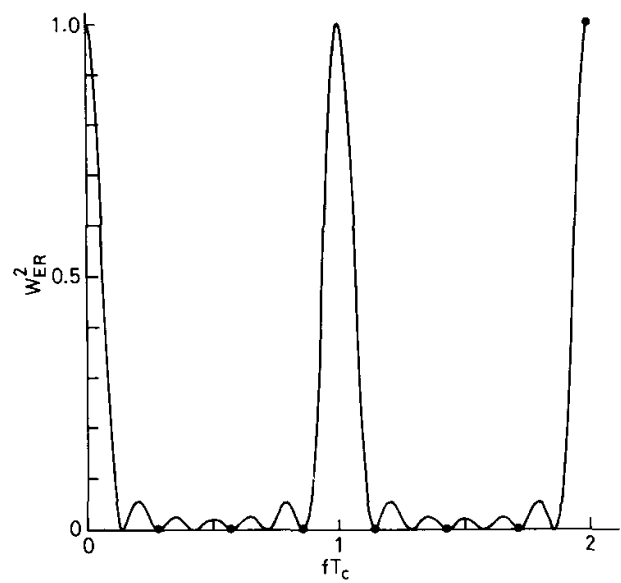

Fig. 10 Weighting function of equally spaced sampling techique and rectangular window with $N=7$ and weighting coefficients (individuated by rounds) in hypothesis of synchronous sampling technique with $u=N$ samples taken in $p=2$ periods of converted signal

zeros. This result does not apply for the other windows previously considered.

\section{Conclusions}

The objective of the criterion described herein has been to compare the different sampling strategies and filtering algorithms used in digital instruments based on nonlinear conversion of the input signals. Obviously the solution for this comparison has been obtained, irrespective of a single observation, a single measurement occasion, or a particular instrument. To this end, the whole of the output signals must be considered for every strategy, avoiding the nuisance contributions of the incidental quantities which can vary from occasion to occasion, from measurement to measurement, and from instrument to instrument, locally conditioning the output signal. The Bayesian approach helps us to incorporate prior information into that variability, enlarging the incidental quantities into corresponding statistical functions whose asymptotic behaviour guarantees the same building block for the comparison analysis. We believe that the quantitative characterisation of the performance function derived from our approach can be improved, but this depends only on the evolution of the sampling and filtering techniques and on the optimisation algorithms; in fact the criterion remains the same because it does not depend on the technical realisations and actual observations.

By using the proposed criterion a general expression for the performance analysis, valid for all the sampling strategies (synchronous, asynchronous and random) considered here, can be deduced; this expression depends on the frequency response of the digital filter used and on the characteristic function of the probability distribution of the random variables generating the sampling instants. Moreover, the particular formulae obtained for all of these sampling strategies, each one using different filtering algorithms, have been discussed. The experimental values of the performance function for different frequencies were found by means of a prototype and were in very good agreement with the theoretical values, confirming the validity of the proposed method.

Finally, it has been shown that in all the asynchronous sampling techniques, the asymptotic mean-squared error allows us to determine the uncertainty of an instrument as a function of the frequency of the converted signal.

\section{References}

1 OPPENHEIM, A.V., and SCHAFER, R.W.: 'Discrete-time signal processing' (Prentice-Hall, NJ, 1989)

2 RABINER, L.R., and GOLD, B.: 'Theory and application of digital signal processing' (Prentice-Hall, NJ, 1975)

3 STRUM, R.D., and KIRK, D.E. 'Discrete systems and digital signal processing' (Addison Wesley, New York, 1988)

4 PAPOULIS, A.: 'Probability, random variables, and stochastic processes' (McGraw-Hill, New York, 1984)

5 Box, G.E.P., and Tiao, G.C.: 'Bayesian inference in statistical analysis' (Addison-Wesley, New York, 1973)

6 TURGEL, R.S.: 'Digital wattmeter using a sampling method', IEEE Trans., 1974, IM-23, (4), pp. 337-341

7 HILL, J.J.: 'Design of a microprocessor-based digital wattmeter', IEEE Trans., 1981, IECI-28, (3), pp. 180-184

8 DIX, C.H.: 'Calculated performance of a digital sampling wattmeter using systematic sampling', IEE Proc. A, 1982, 129, (3), pp. 172-175 9 CLARKE, F.J.J., and STOCKTON, J.R.: 'Principles and theory of wattmeters operating on the basis of regularly spaced sample pairs', J. Phys. E, 1982, 15, pp. 645-652

10 MATOUKA, M.F.: 'A wide-range digital power/energy meter for systems with nonsinusoidal waveforms', IEEE Trans., 1982, IE-29, (1), pp. 18-31

11 FILICORI, F., MIRRI, D., and RINALDI, M.: 'Error estimation in sampling digital wattmeters', IEE Proc. $A, 1985$, 132, (3), pp. $122-128$

12 FILICORI, F., IUCULANO, G., and MIRRI, D.: 'Mixed-mode sampling and error estimation in digital wattmeters', Electron. Lett. $1984,20,(20)$, pp. 828-829

13 STENBAKKEN, G.N.: 'A wideband sampling wattmeter', IEEE Trans., 1984, PAS-103, (10), pp. 2919-2926

14 CORNEY, A.C., and PULLMAN, R.T.: 'Digital sampling laboratory wattmeter', IEEE Trans., 1987, IM-36, (1), pp. 54-59

15 LU ZU-LIANG: "An error estimate for quasi-integer-period sampling and an approach for improving its accuracy', IEEE Trans. 1988, IM-37, (2), pp. 219-222

16 FILICORI, F., IUCULANO, G., MENCHETTI, A., and MIRRI, D.: 'A random asynchronous sampling strategy for measurement instruments based on non-linear signal conversion', IEE Proc. $A$, 1989, 136, (3), pp. 141-150

17 FILICORI, F., IUCULANO, G., MENCHETTI, A., MIRRI, D and CATELANI, M.A.: 'New performance function for the comparison of different sampling strategies in non-linear conversion instruments'. IEEE Instrumentation and Measurement Technology Conference (IMTC/89), Washington, April 1989, pp. 307-311

18 ANDRIA, G., SAVINO, M., and TROTTA, A.: 'Windows and interpolation algorithms to improve electrical measurement accuracy', IEEE Trans. 1989, IM-38, (4), pp. 856-863

19 MIRRI, D., FILICORI, F., IUCULANO, G, and MENCHETTI A.: 'Efficient implementation of digital measurement instruments by A.: 'Efficient implementation of digital measurement instruments by random asynchronous sampling. Proceedings of IMEKO TC4 Symposium 'Measurement in Electrical and Electronic Power
Systems', Zurich, September 1989, pp. 231-240

20 MIRRI, D., FILICORI, F., IUCULANO, G., MENCHETTI, A., and CATELANI, M.A.: 'Accuracy evaluation of digital wattmeters based on different sampling strategies'. Proceedings of IMEKO TC7 Symposium 'Knowledge based measurement', Karlsruhe, September 1990 , pp. 117-124

21 ISO/TAG 4/WG3: 'Guide to the expression of the uncertainty in measurement (sixth draft)'. ISO, Geneve, August 1991

\section{Appendixes}

\subsection{Appendix A}

For the evaluation of the statistical mean, identified by the symbol $\langle\cdot\rangle$, it is necessary to consider the simultaneous random contributions of the variables $k, \tau$ and of the vector $\bar{X}_{k}$. To this end it is convenient to recall the 
definition of the conditional mean assuming $k=k$ and $\tau=\tau$, which we indicate by $M\{\cdot \mid \cdot\}$. In general, for the generic $r$ th-order moment we can write [4],

$$
\begin{aligned}
\left\langle\hat{S}_{k}^{(}\left(\tau, \bar{X}_{k}\right)\right\rangle= & \left\langle M\left\{\hat{S}_{k}^{T}\left(\tau, \bar{X}_{k}\right) \mid k, \tau\right\}\right\rangle \\
= & \frac{1}{2 h+1} \sum_{k=-h}^{+h} \frac{1}{T} \\
& \times \int_{-T / 2}^{+T / 2} M\left\{\hat{S}_{k}^{T}\left(\tau, \bar{X}_{k}\right) \mid k=k, \tau=\tau\right\} d \tau
\end{aligned}
$$

where

$$
\begin{aligned}
& M\left\{\hat{S}_{k}^{T}\left(\tau, \bar{X}_{k}\right) \mid k=k, \tau=\tau\right\} \\
& \quad=\int_{-\infty}^{+\infty} \hat{S}_{k}^{\leftarrow}(\tau, x) f_{X_{k}}(x \mid k=k, \tau=\tau) d x
\end{aligned}
$$

is the $r$-conditional moment assuming $k=k$ and $\tau=\tau$. Obviously this conditional moment, considered as a function of the collection of all the possible couples of values $(k, \tau)$, is itself a random variable and, in this sense, it can be written $\left\langle\boldsymbol{M}\left\{\hat{\boldsymbol{S}}_{\boldsymbol{k}}\left(\tau, \overline{\boldsymbol{X}}_{\boldsymbol{k}}\right) \mid \boldsymbol{k}, \tau\right\}\right\rangle$ as expressed in the first passage of eqn. 46 . To derive eqn. 46 we have taken into account the fact that the random variables $k$ and $\tau$ are independent of each other.

As the generation of the random vector $\bar{X}_{k}$, whose components $\left\{\boldsymbol{X}_{k}, \boldsymbol{X}_{k-1}, \ldots, \boldsymbol{X}_{k-N+1}\right\}$ are random variables distributed in the same interval $(-b,+b)$, is independent of any couple of values $(k, \tau)$, we impose for the conditional density of a generic $X_{k-i}$ assuming $\{\boldsymbol{k}=k, \tau=\tau\}$ the following expression

$$
f_{\mathbf{X}_{k-i}}(x \mid \boldsymbol{k}=k, \tau=\tau)= \begin{cases}f(x) & -b \leqslant x \leqslant+b \\ 0 & \text { elsewhere }\end{cases}
$$

for any $i=0, \ldots, N-1, f(x)$ being the common probability density associated to the random generation. Consequently we obtain for the generic rth-conditional moment

$$
M\left\{\hat{S}_{k}^{\dagger}\left(\tau, \bar{X}_{k}\right) \mid k=k, \tau=\tau\right\}=\left\langle\hat{S}_{k}^{r}\left(\tau, \bar{X}_{k}\right)\right\rangle
$$

$$
r=1,2, \ldots
$$

By substituting eqn. 48 in eqn. 46 we obtain

$$
\left\langle\hat{S}_{k}^{r}\left(\tau, \bar{X}_{k}\right)\right\rangle=\frac{1}{2 h+1} \sum_{k=-h}^{+h} \frac{1}{T} \int_{-T / 2}^{+T / 2}\left\langle\hat{S}_{k}^{r}\left(\tau, \bar{X}_{k}\right)\right\rangle d \tau
$$

where, by recalling eqn. 5 and by assuming

$$
B_{q i}=a_{i} \exp \left(-j \omega_{q} i T_{c}\right)
$$

this results in

$$
\begin{aligned}
\hat{S}_{k}^{\prime}\left(\tau, \bar{X}_{k}\right)=\left[\sum_{i=0}^{N-1} a_{i} S_{o}\right. & +\sum_{\substack{q=-\infty \\
q \neq 0}}^{+\infty} A_{q} \exp \left(j \omega_{q}\left[k T_{c}+\tau\right]\right) \\
& \left.\times \sum_{i=0}^{N-1} B_{q i} \exp \left(j \omega_{q} X_{k-i} T_{c}\right)\right]
\end{aligned}
$$

By imposing $r=1$ and by taking the statistical mean we obtain

$$
\begin{aligned}
\left\langle\hat{S}_{k}\left(\tau, \bar{X}_{k}\right)\right\rangle= & \sum_{i=0}^{N-1} a_{i} S_{o}+\sum_{\substack{q=-\infty \\
q \neq 0}}^{+\infty} A_{q} \exp \left(j \omega_{q}\left[k T_{c}+\tau\right]\right) \\
& \times \Phi\left(\omega_{q} T_{c}\right) \sum_{i=0}^{N-1} B_{q i}
\end{aligned}
$$

where

$$
\begin{aligned}
\Phi\left(\omega_{q} T_{c}\right) & =\left\langle\exp \left(j \omega_{q} X_{k-i} T_{c}\right\rangle\right. \\
& =\int_{-b}^{+b} \exp \left(j \omega_{q} x T_{c}\right) f(x) d x
\end{aligned}
$$

with $\Phi(0)=1$ and $\left|\Phi\left(\omega_{a} T_{c}\right)\right| \leqslant 1$, is the characteristic function, in the normalised frequency (rad) domain $\omega T_{c}$ of every $X_{k-i}$, for $i=0, \ldots, N-1$, and $f(x)$ is the probability density given by eqn. 47 . By substituting eqn. 52 in eqn. 49 with $r=1$, as

$$
\frac{1}{T} \int_{-T / 2}^{+T / 2} \exp \left(j \omega_{q} \tau\right) d \tau=\frac{\sin \left(\omega_{q} T / 2\right)}{\omega_{q} T / 2}=\operatorname{sinc}\left(f_{q} T\right)
$$

and

$$
\begin{aligned}
& \frac{1}{2 h+1} \sum_{k=-h}^{+h} \exp \left(j \omega_{q} k T_{c}\right) \\
& =\frac{1}{2 h+1} \frac{\exp \left(j \omega_{q}(h+1) T_{c}\right)-\exp \left(-j \omega_{q} h T_{c}\right)}{\exp \left(j \omega_{q} T_{c}\right)-1} \\
& =\frac{1}{2 h+1} \frac{\sin \left(\pi f_{q} T_{c}(2 h+1)\right)}{\sin \left(\pi f_{q} T_{c}\right)} \\
& =\frac{\operatorname{sinc}\left(f_{q} T_{c}(2 h+1)\right)}{\operatorname{sinc}\left(f_{q} T_{c}\right)}
\end{aligned}
$$

this results in

$$
\begin{aligned}
\left\langle\hat{S}_{k}\left(\tau, \bar{X}_{k}\right)\right\rangle= & \sum_{i=0}^{N-1} a_{i} S_{o}+\sum_{\substack{q=-\infty \\
q \neq 0}}^{+\infty} A_{q} Z_{h}\left(f_{q}, T_{c}, T\right) \\
& \times \Phi\left(2 \pi f_{q} T_{c}\right) \sum_{i=0}^{N-1} B_{q i}
\end{aligned}
$$

where

$$
Z_{h}\left(f_{q} T_{c}, T\right)=\frac{\operatorname{sinc}\left(f_{q} T_{c}(2 h+1)\right)}{\operatorname{sinc}\left(f_{q} T_{c}\right)} \operatorname{sinc}\left(f_{q} T\right)
$$

Therefore the asymptotic expectation (see eqn. 6) results in

$$
\bar{S}=\lim _{\substack{h \rightarrow \infty \\ T \rightarrow \infty}}\left\langle\hat{S}_{k}\left(\tau, \bar{X}_{k}\right)\right\rangle=\sum_{i=0}^{N-1} a_{i} S_{o}
$$

because the limit of $\operatorname{sinc}\left(f_{\mathrm{a}} T\right)$, with $T$ tending to infinity, is always null. It is important to emphasise that, in the synchronous case, where the signal is periodic with period $T_{1}\left(f_{q}=q f_{1}, q\right.$ being the integer), by assuming $T_{1}=T \operatorname{sinc}\left(f_{q} T\right)$ results identically null, and so $Z_{h}=0$, for any $q$ independently by $h$; hence the expression of eqn. 58 is valid, in this last case, also for the finite expectation.

By substituting eqn. 58 into eqn. 11, and by assuming a scale factor equal to one (see eqn. 6), the result is that the bias $\Delta S_{0}$ is always equal to zero.

To evaluate eqn. 49 for $r=2$, eqn. 50 has to be developed for $r=2$, which has the following form

$$
\begin{aligned}
\hat{S}_{k}^{2}\left(\tau, \bar{X}_{k}\right)= & {\left[\sum_{i=0}^{N-1} a_{i}\right]^{2} S_{o}^{2}+\sum_{\substack{q=-\infty \\
q \neq 0}}^{+\infty} \sum_{\substack{q^{\prime}=-\infty \\
q \neq 0}}^{+\infty} A_{q} A_{q^{\prime}} } \\
& \times \exp \left(j\left[\omega_{q}+\omega_{q^{\prime}}\right]\left[k T_{c}+\tau\right]\right) \\
& \times \sum_{i=0}^{N-1} \sum_{i^{\prime}=0}^{N-1} B_{q i} B_{q^{\prime} i} \\
& \times \exp \left(j\left[\omega_{q} \bar{X}_{k-i}+\omega_{q^{\prime}} X_{k-i^{\prime}}\right] T_{c}\right) \\
& +2 \sum_{i=0}^{N-1} a_{i} S_{o}\left[\hat{S}_{k}\left(\tau, \bar{X}_{k}\right)-\sum_{i=0}^{N-1} a_{i} S_{o}\right]
\end{aligned}
$$


To evaluate the statistical mean of this equation, we can recall eqn. 53 and that

$$
\sum_{i=0}^{N-1} \sum_{\substack{i^{\prime}=0 \\ i \neq i^{\prime}}}^{N-1}=\sum_{i=0}^{N-1} \sum_{i^{\prime}=0}^{N-1}-\sum_{\substack{i=0 \\ i=i^{\prime}}}^{N-1}
$$

Furthermore we have to take into account that for $i=i^{\prime}$, this results in $\boldsymbol{X}_{k-i} \equiv \boldsymbol{X}_{\boldsymbol{k}-i^{\prime}} ;$ instead, $\boldsymbol{X}_{\boldsymbol{k}-\boldsymbol{i}}$ and $\boldsymbol{X}_{\boldsymbol{k}-\boldsymbol{i}^{\prime}}$ resulting independently for $i \neq i^{\prime}$, we have

$$
\begin{gathered}
\left\langle\exp \left(j 2 \pi f_{q}\left[X_{k-i}+X_{k-i^{\prime}}\right] T_{c}\right\rangle\right. \\
=\Phi\left(2 \pi f_{q} T_{c}\right) \Phi\left(2 \pi f_{q^{\prime}} T_{c}\right)
\end{gathered}
$$

Therefore this results in

$$
\begin{aligned}
& \left\langle\hat{S}_{k}^{2}\left(\tau, \bar{X}_{k}\right)\right\rangle \\
& =\left[\sum_{i=0}^{N-1} a_{i}\right]^{2} S_{o}^{2}+\sum_{\substack{q=-\infty \\
q \neq 0}}^{+\infty} \sum_{\substack{q^{\prime}=-\infty \\
q \neq 0}}^{+\infty} A_{q} A_{q^{\prime}} \\
& \quad \times \exp \left(j\left[\omega_{q}+\omega_{q^{\prime}}\right]\left[k T_{c}+\tau\right]\right) \\
& \quad \times \quad \Phi\left(\left[\omega_{q}+\omega_{q}\right] T_{c}\right) \sum_{i=0}^{N-1} B_{q i} B_{q^{\prime} i^{\prime}} \\
& \left.\quad+\Phi\left(\omega_{q} T_{c}\right) \Phi\left(\omega_{q^{\prime}} T_{c}\right) \sum_{i=0}^{N-1} \sum_{\substack{i^{\prime}=0 \\
i \neq i^{\prime}}}^{N-1} B_{q i} B_{q^{\prime} i^{\prime}}\right] \\
& \quad+2 \sum_{i=0}^{N-1} a_{i} S_{o}\left[\hat{S}_{k}\left(\tau, \bar{X}_{k}\right)-\sum_{i=0}^{N-1} a_{i} S_{o}\right]
\end{aligned}
$$

By substituting eqn. 62 in eqn. 49 with $r=2$ and by recalling eqns. 54,55 and 57 , we obtain

$\left\langle\hat{S}_{k}^{2}\left(\tau, \bar{X}_{k}\right)\right\rangle$

$$
\begin{aligned}
& =\left[\sum_{i=0}^{N-1} a_{i}\right]^{2} S_{o}^{2} \\
& +\sum_{\substack{q=-\infty \\
q \neq 0}}^{+\infty} \sum_{\substack{q^{\prime} \\
q \neq 0}}^{+\infty} A_{q} A_{q^{\prime}} Z_{h}\left(\left[f_{q}+f_{q^{\prime}}\right], T_{c}, T\right) \\
& \quad \times\left[\Phi\left(2 \pi\left[f_{q}+f_{q^{\prime}}\right] T_{c}\right) \sum_{i=0}^{N-1} B_{q i} B_{q^{\prime} i}\right. \\
& \left.\quad+\Phi\left(2 \pi f_{q} T_{c}\right) \Phi\left(2 \pi f_{q^{\prime}} T_{c}\right) \sum_{i=0}^{N-1} \sum_{\substack{i^{\prime}=0 \\
i \neq i^{\prime}}}^{N-1} B_{q i} B_{q^{\prime} i^{\prime}}\right] \\
& +2 \sum_{i=0}^{N-1} a_{i} S_{o}\left[\left\langle\hat{S}_{k}\left(\tau, \bar{X}_{k}\right)\right\rangle-S_{o} \sum_{i=0}^{N-1} a_{i}\right]
\end{aligned}
$$

By considering the limit of eqn. 63 when $h$ and $T$ tend to the infinite, the contribution of the harmonics to the double sum is not null only when $f_{q}=-f_{q^{\prime}}=f_{-q^{\prime}}$; consequently $A_{q^{\prime}}=A_{q}^{*}$ and $B_{q^{\prime} i}=B_{q i}^{*}$ and $B_{q^{\prime} i^{\prime}}=B_{q i^{\prime}}^{*}$. Therefore the result is

$$
\begin{aligned}
\lim _{\substack{h \simeq \infty \\
T \simeq \infty}}\left\langle\hat{S}_{k}^{2}\left(\tau, \bar{X}_{k}\right)\right\rangle & \\
= & {\left[\sum_{i=0}^{N-1} a_{i} S_{0}^{2}\right]+2 \sum_{q=1}^{\infty}\left|A_{q}\right|^{2} } \\
& \times\left[\sum_{i=0}^{N-1} a_{i}^{2}+\left|\Phi\left(2 \pi f_{q} T_{c}\right)\right|^{2}\right. \\
& \left.\times\left(\left|\sum_{i=0}^{N-1} B_{q i}\right|^{2}-\sum_{i=0}^{N-1} a_{i}^{2}\right)\right]
\end{aligned}
$$

as $\left|B_{q i}\right|^{2}=a_{i}^{2}$ (see eqn. 50).

IEE PROCEEDINGS-A, Vol. 139, No. 4, JULY 1992
By recalling eqn. 12 and by substituting eqn. 64 we obtain

$$
\begin{aligned}
\sigma^{2}=2 \sum_{q=1}^{\infty}\left|A_{q}\right|^{2}\left[\sum_{i=0}^{N-1} a_{i}^{2}\right. & +\left|\Phi\left(2 \pi f_{q} T_{c}\right)\right|^{2} \\
& \left.\times\left(\left|\sum_{i=0}^{N-1} B_{q i}\right|^{2}-\sum_{i=0}^{N-1} a_{i}^{2}\right)\right]
\end{aligned}
$$

By introducing the normalised frequency response of the filter FIR defined by eqn. 4 , i.e.

$$
H\left(2 \pi f T_{c}\right)=\sum_{i=0}^{N-1} a_{i} \exp \left(-j 2 \pi f i T_{c}\right)
$$

we obtain from eqn. 51

$$
\sum_{i=0}^{N-1} B_{q i}=H\left(2 \pi f_{q} T_{c}\right)
$$

Therefore eqn. 65 can be rewritten as follows

$$
\sigma^{2}=2 \sum_{q=1}^{\infty}\left|A_{q}\right|^{2} W^{2}\left(f_{q} T_{c}\right)
$$

with $W^{2}\left(f_{q} T_{c}\right)$ defined by

$$
\begin{aligned}
W^{2}\left(f_{q} T_{c}\right)= & \sum_{i=0}^{N-1} a_{i}^{2}+\left|\Phi\left(2 \pi f_{q} T_{c}\right)\right|^{2} \\
& \times\left(\left|H\left(2 \pi f_{q} T_{c}\right)\right|^{2}-\sum_{i=0}^{N-1} a_{i}^{2}\right)
\end{aligned}
$$

\subsection{Appendix B}

The weighting function for the equally spaced sampling and the rectangular window (see eqn. 18)

$$
W_{E R}^{2}\left(f T_{c}\right)=\frac{1}{N^{2}} \frac{\sin ^{2} N \pi f T_{c}}{\sin ^{2} \pi f T_{c}}
$$

is periodic with respect to $f T_{c}$ with a period equal to 1 . The frequencies at which this function reaches its minima are determined by the following $(N-1)$ values

$$
f_{i \min } T_{c}=\frac{i}{N} \quad \text { with } i=1, \ldots,(N-1)
$$

where the function $\sin N \pi f T_{c}$ is null; therefore $W_{E R}^{2}\left(f_{i \min } T_{c}\right)=0$. To derive the frequencies $f_{r \text { max }} T_{c}$ which maximise the weighting function, it should be noted that $\log W_{E R}^{2}\left(f T_{c}\right)$ and $W_{E R}^{2}\left(f T_{c}\right)$ attain their maxima at the same values. Taking the logarithm of eqn. 70 , deriving with respect to $f$ and equating to zero, we obtain

$$
N \operatorname{tg} \pi f T_{c}=\operatorname{tg} N \pi f T_{c}
$$

It can be checked that the solutions to the above equations effectively maximise the performance function; to this end we consider the second derivative of eqn. 70 and verify that it is negative. The final inequality obtained is the following

$$
\sin ^{2} N \pi f T_{c}-N^{2} \sin ^{2} \pi f T_{c}<0
$$

or equivalently

$$
\frac{t g^{2} N \pi f T_{c}}{1+t g^{2} N \pi f T_{c}}-N^{2} \frac{t g^{2} \pi f T_{c}}{1+t g^{2} \pi f T_{c}}<0
$$

By introducing eqn. 72 in eqn. 74 , the latter is satisfied identically.

By using Euler's formulas it can be shown that eqn. 72 is equivalent to

$$
\sin \left[(N+1) \pi f T_{c}\right]=\frac{N+1}{N-1} \sin \left[(N-1) \pi f T_{c}\right]
$$


In the hypothesis that $N$ is sufficiently large, so that $(N+1) /(N-1) \simeq 1$, eqn. 75 can be solved with an acceptable approximation by

$$
f_{i \max } T_{c}=\left(i+\frac{1}{2}\right) \frac{1}{N} \quad \text { with } i=1, \ldots, N
$$

The corresponding values of the performance function, i.e. their maxima, are given by

$$
W_{E R}^{2}\left(f_{i \max } T_{c}\right)=\frac{1}{N^{2}} \frac{1}{\sin ^{2}\left(\left[i+\frac{1}{2}\right] \frac{\pi}{N}\right)}
$$

\subsection{Appendix C}

We must introduce the weights defined by eqn. 24 into eqn. 16 to derive the frequency response of a digital filter realised with a trapezoidal window with clipped ends. Consequently, by imposing

$$
q=e^{-j \omega T_{c}}
$$

we obtain

$$
\begin{aligned}
m n H\left(\omega T_{c}\right)= & \sum_{i=0}^{n-1}(i+1) q^{i}+n \sum_{i=n}^{m-2} q^{i} \\
& +\sum_{i=m-1}^{m+n-2}(m+n-i-1) q^{i}
\end{aligned}
$$

If we change the index $i$ to $i-m+1$ in the last sum after simple manipulation this results in

$$
\begin{aligned}
m n H\left(\omega T_{c}\right)= & \left(1+n q^{m-1}\right) \sum_{i=0}^{n-1} q^{i}+n \sum_{i=n}^{m-2} q^{i} \\
& +\left(1-q^{m-1}\right) \sum_{i=0}^{n-1} i q^{i}
\end{aligned}
$$

By remembering the properties of the geometric and the arithmetic geometric progressions and simplifying, we obtain

$$
\begin{aligned}
m n H\left(\omega T_{c}\right)= & \left(1+n q^{m-1}\right) \frac{q^{n}-1}{q-1}+n \frac{q^{m-1}-q^{n}}{q-1} \\
& +\left(1-q^{m-1}\right)\left[\frac{(n-1) q^{n}}{q-1}+\frac{q\left(1-q^{n-1}\right)}{(q-1)^{2}}\right] \\
= & \frac{\left(q^{m}-1\right)\left(q^{n}-1\right)}{(q-1)^{2}}
\end{aligned}
$$

Finally, by recalling eqn. 78 , this results in

$$
\begin{aligned}
& H_{T}\left(2 \pi f T_{c}\right) \\
& \quad=\frac{1}{m n} \frac{\left(\exp \left(-j \omega m T_{c}\right)-1\right)\left(\exp \left(-j \omega n T_{c}\right)-1\right)}{\left(\exp \left(-j \omega T_{c}\right)-1\right)^{2}} \\
& =\frac{1}{m n} \exp (-j \pi(m+n-2)) \frac{\sin \left(\pi m f T_{c}\right) \sin \left(\pi n f T_{c}\right)}{\sin ^{2}\left(\pi f T_{c}\right)} \\
& =\exp (-j \pi(m+n-2)) \frac{\operatorname{sinc}\left(m f T_{c}\right) \operatorname{sinc}\left(n f T_{c}\right)}{\operatorname{sinc}^{2}\left(f T_{c}\right)}
\end{aligned}
$$

Now, if we remember that the frequency response in the case of a rectangular window is given by eqn. 17, we can write

$$
H_{T}\left(2 \pi f T_{c}\right)=H_{R n}\left(2 \pi f T_{c}\right) H_{R m}\left(2 \pi f T_{c}\right)
$$

where at the second member we have introduced a second index to distinguish the length of the rectangular window. Eqn. 83 shows [3] that, instead of a trapezoidal window with clipped ends, we can use the cascade of two rectangular windows. 